\title{
29 Year Old Man with Multiple Sclerosis and Schizophrenia: A Case Report
}

\author{
Nahid Noorani ${ }^{1}$, Fateme Hadi², Hamid Reza Ahmadkhaniha ${ }^{3}$
}

\author{
${ }^{1}$ M.D., Psychiatrist, Tehran, Iran \\ ${ }^{2}$ M.D., Assistant Professor of Psychiatry, Mental Health Research Center, Tehran Institute of Psychiatry, School of \\ Behavioral Sciences and Mental Health, Iran University of Medical Sciences, Tehran, Iran \\ ${ }^{3}$ MD; Professor of Psychiatry, Department of Psychiatry, Iran University of Medical Science, Tehran, Iran
}

\section{Type of article: Case report}

\begin{abstract}
Multiple sclerosis (MS) is the most common debilitating neurological disease that affects adults, whether young adults or middle-aged. Although, most attention is toward the neurological signs of the disease, the neuropsychiatric signs are not uncommon. This case report presents a 29 year old male with a record of obsessive-compulsive disorder (OCD) without psychotic disorder, which coincides with the diagnosis MS, has been stricken to auditory hallucinations and reference delusion. The patient received some antipsychotic drugs such as Haloperidol and Perphenazine irregularly, but any psychotic signs of the patient were never in control. During this period he had several active episodes of MS disease, wherein the symptoms had subsided due to hospitalization and received corticosteroids pulse. The first time the patient was submitted to the emergency unit of Rasoul Akram Hospital, there was the possibility of schizophrenia which was confirmed in subsequent visits. The signs of the patient were not controllable for a long time and finally fully controlled by a combination of Aripiprazole (abilizol), Risperidone and Sertraline, and currently, for almost 3 years, both psychotic symptoms and MS disease have been under control. Our patient seems to catch the MS disease and schizophrenia simultaneously. There was no relation between MS and psychosis episodes and the MS attacks. Since the onset the patient had several acute MS attacks of MS, and hospitalization several times. These findings and characteristics regarding our patient made him completely different from other reported cases of MS along with neuropsychiatric signs which may help doctors in diagnosis and managment of similar cases.

Keywords: Multiple sclerosis (MS), Schizophrenia, Aripiprazole (Abilizol), Risperidone, Avonex
\end{abstract}

\section{Introduction}

Multiple sclerosis (MS) is a chronic demyelinative disease, which is specified by the patient's focal neurological damage (1). It can involve each part of CNS such as hemispheres of the brain, optic nerve, cerebellum, brain stem and spinal cord (2-5). Most people between the ages of 20 to 40 years old are involved, and its prevalence in women is more than in men. The prevalence of MS disease in different regions, is different, and most prevalence is in Western Europe and the United States (3-5). The neuropsychiatric signs in MS disease are not uncommon, with symptoms such as depression, anxiety, irritability, apathy and psychosis. Depression is the most common neuropsychiatric sign $(2,6)$. In cases of associating neuropsychiatric signs together with MS, patients' functions become weaker (2). Psychosis prevalence in MS in different studies, were different to some extent and is approximately 2 to 3 percent. $(1,7)$ It is said that in some studies, the rarest neuropsychiatric sign in MS is hallucination and delusion (2). Information on co morbidity of schizophrenia and MS is very low. Neuropsychiatric symptoms may be seen gradually in the course of MS, but it's unusual at the onset (2-3.2\%) (7). In this case the psychotic symptoms and MS started simultaneously, in fact the patient catches MS and schizophrenia at the same time. So, following the course of both of these diseases, their effects on each other, rejection of organic causes and possible causes of psychosis were important to us.

\section{Corresponding author:}

Hamid Reza Ahmadkhaniha, Department of Psychiatry, Iran University of Medical Science, Tehran, Iran. Tel: +98.9123546015, E-mail: dr_ahmadkhaniha@yahoo.com Received: November 09, 2015, Accepted: July 24, 2016, Published: December 2016 iThenticate screening: January 28, 2016, English editing: September 14, 2016, Quality control: October 26, 2016 (C) 2016 The Authors. This is an open access article under the terms of the Creative Commons Attribution-NonCommercialNoDerivs License, which permits use and distribution in any medium, provided the original work is properly cited, the use is non-commercial and no modifications or adaptations are made. 


\section{Case presentation}

The patient was a 29 year old male, with no personal or family record of psychotic illness. First time was 9 years ago when the patient was 20 years old when he had suddenly been stricken by diplopia and numbness in the left side of his forehead, and for the first time, he was hospitalized on the neurology ward at a general hospital. At the same time he had symptoms such as delusional thoughts and grandiosity (he thought he was telepathic, and able to read others' thoughts). In neurological examinations, the diplopia of both eyes and the left half of his face, paresthesia was significant. MRI scan showed obvious white matter signal changes especially surrounding the ventricles and left parieto-occipital lobe.

Also, in the cervical MRI, the demyelinative spine plaques were seen to have accumulated more in the upper part, about the level C2-C3. After diagnosing the MS, corticosteroids pulse corticosteroid therapy for the patient was started. After 5 days neurological symptoms subsided and the patient with a medication order of Avonex (100INFB) was discharged. For 3 years, the patient still had the psychotic symptoms and these symptoms never subsided. During this period the patient experienced 5 episodes of MS acute attacks. In March 2007 the patient along with his father visited the psychiatric emergency unit of Rasoul-e-Akram hospital for the first time. He was a 23 year old freshman and student of mechanics. He had signs such as auditory hallucinations, reference and grandiosity delusion. He also had symptoms of obsession with washing and cleaning. By his family explanations and other signs, possibility of schizophrenia or organic psychosis for the patient was confirmed and suggested to the patient. Risperidone $4 \mathrm{mg}$ and Citalopram $20 \mathrm{mg}$ were prescribed and they were recommended to recourse after two weeks. But it took 3 years for the patient's next visit, at which time he was taking medication irregularly. This time the patient was admitted to the Iran Psychiatric Hospital. He was already 26 years old. During this time the patient was referred to several psychiatrists and was prescribed various medications which he had not taken regularly. At that time he was taking $8 \mathrm{mg}$ Risperidone, $4 \mathrm{mg}$ Biperiden and 200mg Sertraline. Because of his medical history there were signs of extra pyramidal and ochologiric crisis in the patient's medical history, and the Biperiden dose was increased to $6 \mathrm{mg}$. In additional medical history the following information was obtained:

There were no other disorders except MS in medical history, he had no acute attack for about a year and took Betaferon for controlling his MS disease. In past psychiatric record history, the first signs of obsession with washing and controlling began for the first time in a period before a university entrance exam, which had disappeared after a while without any specific treatment. In the patient's family record history, his mother had washing and cleaning obsession symptoms, but she didn't take any medication. He has 2 brothers and 1 sister, (One of his brothers was his identical twin). His twin brother, who had finished his education, had an M.S. degree in industrial engineering with a master of sciences degree, was employed, and has no medical problem. Other siblings were also healthy, and there was no history of psychotic disorder recorded in the family. On subsequent visits, some of the symptoms of aggression and self-laughing had decreased, but there was still a degree of delusions and hallucinations. Finally, by adding 30mg Abilify, 8mg Risperidone, 200mg Sertraline and 4mg Biperiden, the patient's symptoms were controlled. After 3 years, the patient's psychotic symptoms and obsession were under controll by taking medicine and he had no MS acute attacks. The MRI which the patient had 2012, had no significant change compared to his 2007 MRI, regarding to con-trolling his symptoms, and absence of any MS acute attacks, his MS is non-progressive

\section{Discussion}

Our patient seems to catch the MS disease and schizophrenia simultaneously. There was no relation between MS and psychosis episodes. Since the onset, the patient has had several acute MS attacks and hospitalizations several times. It is almost 3 years since the patient's psychotic symptoms and obsession along with his MS attacks have been under control. According to the analysis of our patient, the first thing that that probably could have been suggested was that the psychotic symptoms could be part of the symptoms of MS. As mentioned before, the neuropsychiatric signs were common in MS patients. However the prevalence of psychotic symptoms were less, compared to other neuropsychiatric symptoms (2). It is an uncommon occurrence at the beginning of the MS disease.(7). In most articles, it had been mentioned that the psychotic symptoms in MS were associated with medical temporal lobe lesions especially in the left -side $(8,9)$. In our case, the accumulation of lesions was at the left hemisphere and parieto-occipital lobe. In most cases of neuropsychiatric signs of MS, for example, by simultaneous treatment and control of inflammatory lesions of MS symptoms and prescribing antipsychotic, we expect the symptoms revolved. But in our case, there was no relation between psychotic symptoms and MS attack episodes and despite controlling and subsidence of MS attacks, psychotic symptoms still persist (Due to neurological symptoms and MRI findings, the diagnosis of MS was strongly considered for patients). In addition to MS, the diagnosis which 
was considered for our patients was schizophrenia (early psychosis). But there were some points which were opposed in initial diagnosis. First, there was no family record of psychosis. The premorbid medical history of the patient is not in favor of schizophrenia, and in this period the patient has no negative signs. Most importantly, the patient has an identical twin brother who does not have any medical or psychiatric disease. Although we know that the extent of co morbidity in monozygotic twins, the concordance rate for schizophrenia is 50 to 60 percent and for MS is $18.2 \%$ which is also reported up to 25 to 30 percent (11). The content of patient delusions had never been unusual.

\section{Conclusions}

Our patient seems to $\mathrm{s}$ catch the MS disease and schizophrenia simultaneously. There was no relation between MS and psychosis attack episodes and MS. Since the onset, the patient had several acute MS attacks, and hospitalizations several times. The findings and characteristics of our patient made him different from other reported cases of MS along with neuropsychiatric signs which may help doctors in diagnosis and management of similar cases.

\section{Acknowledgments:}

We thank Dr. Adnan Tizmaghz for assistance in revision of this paper.

\section{Conflict of Interest:}

There is no conflict of interest to be declared.

\section{Authors' contributions:}

All authors contributed to this project and article equally. All authors read and approved the final manuscript.

\section{References:}

1) Yadav R, Zigmond AS. Temporal lobe lesions and psychosis in multiple sclerosis. BMJ Case Rep. 2010; 2010. pii: bcr0120102651. doi: 10.1136/bcr.01.2010.2651. PMID: 22789690, PMCID: PMC3030118.

2) Figved N, Klevan G, Myhr KM, Glad S, Nyland H, Larsen JP, et al. Neuropsychiatric symptoms in patient with multiple sclerosis. Acta Psychiatr Scand. 2005; 112(6): 463-8. doi: 10.1111/j.16000447.2005.00624.x. PMID: 16279876.

3) Larsen JP, Aarli JA, Nyland H, Riise T. Western Norway, a high-risk area for multiple sclerosis: a prevalence/incidence study in the county of Hordaland. Neurology. 1984; 34(9): 1202-7. PMID: 6540405.

4) Celius EG, Vandik B. Multiple sclerosis in oslo, Norway: Prevalence on 1 January 1995 and incidence over a 25-year period. Eur J Neurol. 2001; 8(5): 463-9. PMID: 11554910.

5) Dahl OP, Aarseth JH, Myhr KM, Nyland H, Midgard R. Multiple sclerosis in Nord-Trøndelag County, Norway: a prevalence and incidence study. Acta Neurol Scand. 2004; 109(6): 378-84. doi: 10.1111/j.16000404.2004.00244.x. PMID: 15147459.

6) Goeb JL, Even C, Nicolas G, Gohier B, Dubas F, Garré JB. Psychiatric side effects of interferon- B in multiple sclerosis. Eur Psychiatry. 2006; 21(3): 186-93. doi: 10.1016/j.eurpsy.2005.09.013. PMID: 16386408.

7) Psychiatric symptoms According multiple sclerosis onset: A case Report. J Neuropsychiatry clin Neurosci. 2014; 26: 2 .

8) Reiss JP, Sam D, Sareen J. Psychosis in multiple sclerosis associated with left temporal lobe lesions on serial MRI scans. J Clin Neurosci. 2006; 13(2): 282-4. doi: 10.1016/j.jocn.2005.02.017. PMID: 16503492.

9) Feinstein A. Neuropsychiatric syndromes associated with multiple sclerosis. J Neurol. 2007; 254(2): II73-6. doi: 10.1007/s00415-007-2017-2. PMID: 17503134.

10) Moghtaderi A, Rakhshanizadeh F, Shahraki-Ibrahimi S. Incidence and prevalence of multiplesclerosis in southeastern Iran. Clin Neurol Neurosurg. 2013; 115(3): 304-8. doi: 10.1016/j.clineuro.2012.05.032. PMID: 22717599.

11) O'Gorman C, Lin R, Stankovich J, Broadley SA. Modelling gentic susceptibility to multiplesclerosis with family data. Neuroepidemiology. 2013; 40(1): 1-12. doi: 10.1159/000341902. PMID: 23075677. 